\title{
Diagnostic value of cardiac computed tomography angiography in patients with left bundle branch block and abnormal single-photon emission computed tomography findings
}

\author{
Ahmed Fathala MD¹, Fatima AL-Wusibai MD², Mohamed Shoukri PhD ${ }^{3}$, Bander Al-Ghamdi MD²
}

\begin{abstract}
A Fathala, F AL-Wusibai, M Shoukri, B Al-Ghamdi. Diagnostic value of cardiac computed tomography angiography in patients with left bundle branch block and abnormal single-photon emission computed tomography findings. Curr Res Cardiol 2015;2(4):157-160.
\end{abstract}

BACKGROUND: The presence of septal perfusion defect on myocardial perfusion single-photon emission computed tomography (SPECT) in patients with known left bundle branch block (LBBB) can present a major diagnostic dilemma.

METHODS: Thirty-nine patients with known LBBB underwent both SPECT and coronary computed tomography angiography (CCTA), a median of 65 days after SPECT. Patients underwent SPECT; CCTA was performed on the basis of abnormal SPECT and presence of clinical symptoms. Exclusion criteria included a history of myocardial infarction, previous coronary artery bypass surgery or percutaneous coronary intervention. RESULTS: Of the study population $(\mathrm{n}=39), 22(56 \%)$ patients were female and the mean $( \pm S D)$ age was $63 \pm 13$ years; all patients were known

to have LBBB diagnosed by electrocardiogram. SPECT results showed 24 (61\%) patients had a reversible septal defect and $15(39 \%)$ had a fixed septal defect. CCTA revealed that 27 (69\%) patients had normal coronary artery or nonobstructive coronary artery disease (CAD); only $12(31 \%)$ had abnormal coronary artery ( $>50 \%$ luminal stenosis). There was no relationship between patterns of SPECT perfusion abnormalities and CCTA results.

CONCLUSION: The presence of abnormal SPECT in patients with LBBB did not necessarily indicate the presence of CAD. Approximately $70 \%$ of patients with LBBB and abnormal SPECT had normal CCTA. The authors strongly believe CCTA must be considered the first noninvasive test in patients without history of CAD and LBBB. Normal CCTA excludes CAD, but patients with obstructive CAD $>50 \%$ on CCTA will benefit from conventional coronary angiography.

Key Words: Abnormal SPECT; CAD; LBBB; Noninvasive cardiac imaging; SPECT

Spontaneous left bundle branch block (LBBB) is associated with $\checkmark$ increased cardiovascular-related overall mortality (1-3). LBBB is characterized by a delay in electrical activity and, accordingly, mechanical activation of the left ventricle resulting in intra- and interventricular asynchrony eventually leading to systolic and diastolic dysfunction $(4,5)$. LBBB results in a septal perfusion defect on myocardial perfusion single-photon emission computed tomography (SPECT), which may be mistaken for myocardial infarction or ischemia depending on whether the defect is fixed or reversible, respectively. The presence of septal perfusion defect in patients with known $\mathrm{LBBB}$ can present a major diagnostic dilemma; differentiation between coronary artery disease (CAD) versus artefact-related LBBB as the etiology of septal defects cannot be established in many cases and invasive coronary angiography may be needed. Cardiac computed tomography angiography (CCTA) is a promising noninvasive technique to detect and characterize stenotic and nonstenotic CAD (6-12). Recent reports have shown that CCTA has excellent diagnostic accuracy in patients with known LBBB. A normal CCTA in this clinical setting is a robust tool to act as a filter and avoid invasive diagnostic procedures. The aim of present study was to evaluate CCTA and abnormal SPECT findings in patients with LBBB.

\section{Study population}

\section{METHODS}

From November 2011 to May 2015, 39 subjects with known LBBB underwent SPECT followed by CCTA (within a median of 65 days). Patients underwent SPECT on appropriate clinical indications. CCAC was performed either on the basis of abnormal SPECT or the presence of clinical symptoms. Exclusion criteria included a history of myocardial infarction, previous coronary artery bypass surgery or percutaneous coronary intervention, previous cardiomyopathy or known valvular heart disease. The study was approved by the authors' hospital review board.

\section{SPECT acquisition and analysis}

Patients underwent rest-stress myocardial perfusion studies with either a separate-day protocol or a same-day stress-rest sequence. The choice of tracer and same-day or separate-day protocol was based on logistic requirements. The rest dose in patients who underwent a separate-day rest-stress protocol was $10 \mathrm{MBq}$ to $370 \mathrm{MBq}$ of either technetium-99 (Tc-99m) sestamibi or tetrofosmin. The stress dose in patients who underwent the rest-stress same-day protocol was $1100 \mathrm{MBq}$ of either Tc-99m sestamibi or tetrofosmin. Tc-99m sestamibi or Tc-99m tetrofosmin was injected during peak pharmacological vasodilation with adenosine $(140 \mu \mathrm{g} / \mathrm{kg} / \mathrm{min})$ or dipyridamole. SPECT imaging was started $30 \mathrm{~min}$ following pharmacological vasodilation. Rest SPECT myocardial perfusion imaging was initiated at approximately $60 \mathrm{~min}$ following injection. SPECT imaging was performed with line source attenuation correction at $90^{\circ}$ dual-detector gamma camera (Cardio MD, Philips Medical System, USA) equipped with attenuation correction and truncation compensation. The acquisition parameters and post processing were performed according to the most recent guidelines of the American Society of Nuclear Cardiology for nuclear cardiology procedures (13).

All images were reoriented in short, vertical and horizontal views using Auto SPECT (Cedars-Sinai Medical Center, Los Angeles, California) for visual interpretation by an experienced nuclear medicine physician. The reader was not biased by clinical information.

${ }^{1}$ Medical Imaging Service, King Faisal Specialist Hospital Eु Research Center; ${ }^{2}$ King Faisal Heart Center; ${ }^{3}$ Department of Cell Biology and National Biotechnology Center, King Faisal Specialist Hospital and Research Center, Riyadh, Saudi Arabia

Correspondence: Dr Ahmed Fathala, King Faisal Specialist Hospital and Research Center, Medical Imaging Service, PO Box 3354, Riyadh, Saudi Arabia. Telephone 9661 552532402, fax 9661 44224841, e-mail ahm35799@hotmail.com 


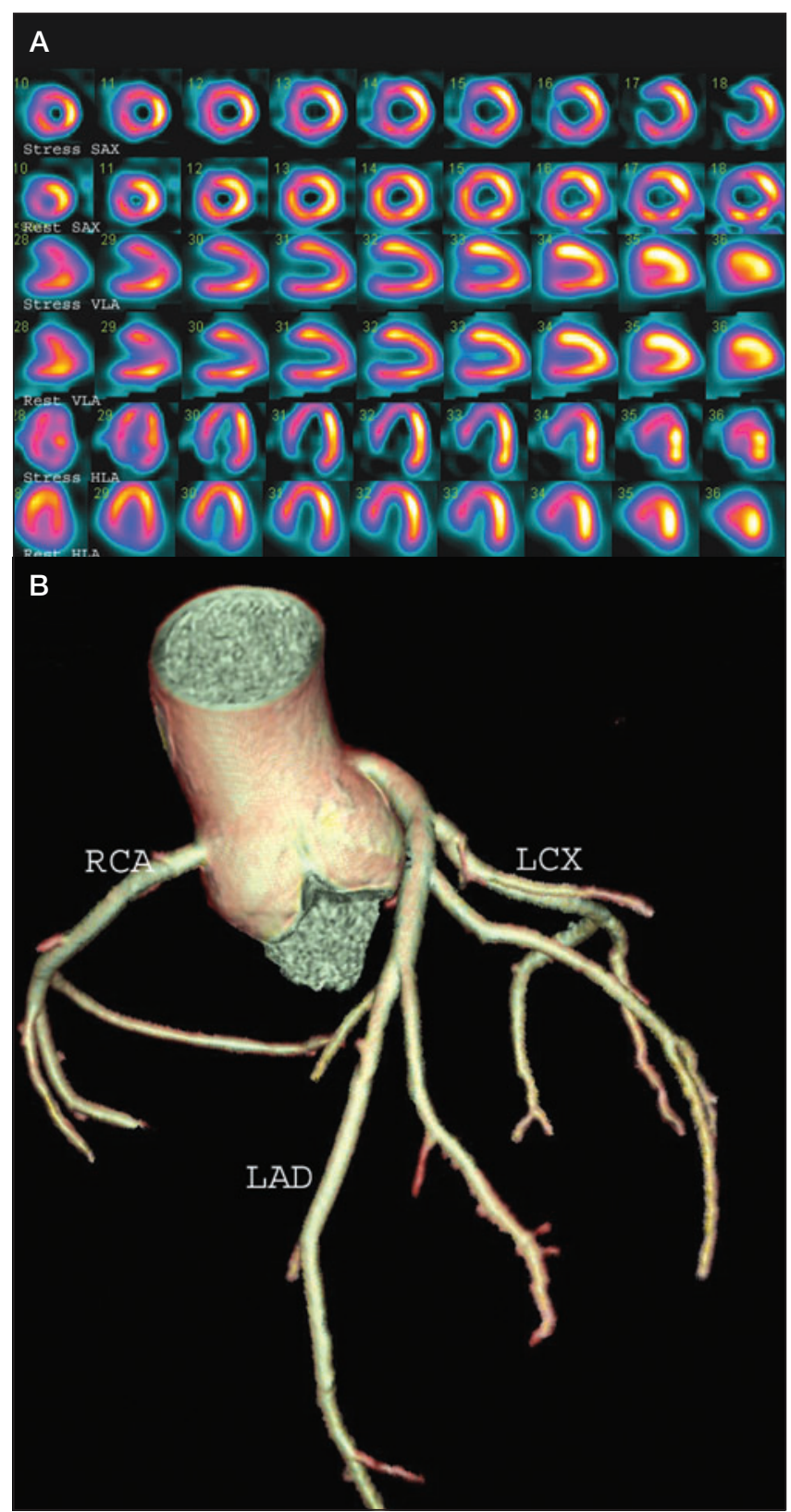

Figure 1) A Dipyridamole stress and rest myocardial perfusion single-photon emission computed tomography in a patient with known left bundle branch block, showing typical a stress-induced septal perfusion abnormality that reverses on rest images. HLA Horizontal long axis; SAX Short axis; VLA Vertical long axis. B Coronary computed tomography of the same patient showed normal coronary artery. LAD Left anterior descending artery; LCX Left circumflex artery; RCA Right coronary artery

Stress and rest perfusion images were scored using 17 tomographic segments, which included six segments each for the basal and midventricular slices, and four segments for the apical short-axis slices. The final segment is located on the most apical part of the left ventricle (14). Finally, gated short-axis images were processed with quantitative SPECT software to measure the ejection fraction. In the visual analysis, the 17 segments were scored for perfusion defects on a four-point system $(0=$ normal; $1=$ mild; $2=$ moderate; and $3=$ severe $)$ for both the stress and rest images. From this analysis, ischemia was defined as a change in segmental score between stress and rest. Segments with no change between stress and rest were classified as nonreversible. Summed stress and rest scores were calculated by summing the 17 segmental scores in each imaged set. Utilizing the summed difference score, defect reversibility was calculated from the difference between the summed stress and rest scores. Summed difference scores $<2$ were considered as no ischemia; 2 to $4=$ mild ischemia; 5 to $8=$ moderate ischemia; and $>8=$ severe ischemia. The reader made the final determination of an abnormal SPECT study by comparing both the perfusion and functional data (Figure 1 represents a typical example of septal perfusion abnormalities in patient with LBBB).

\section{CCTA}

Patients received metoprolol (targeting a heart rate of $\leq 65$ beats $/ \mathrm{min}$ ) and nitroglycerin $0.8 \mathrm{mg}$ sublingually before image acquisition. A bolus tracking technique was used to calculate the time interval between intravenous contrast (Visipaque 320, GE Healthcare, USA) infusion and image acquisition. Final images were acquired using a triphasic protocol (100\% contrast, $40 \% / 60 \%$ contrast/saline and $40 \mathrm{~mL}$ saline). The contrast volume and infusion rate $(5 \mathrm{~mL} / \mathrm{s}$ to $6 \mathrm{~mL} / \mathrm{s}$ ) were individualized according to scan time and patient body habitus. Retrospective electrocardiogram (ECG)-gated data sets were acquired with the GE high-definition CT (GE Healthcare, USA) with $64 \mathrm{~mm} \times 0.625 \mathrm{~mm}$ slice collimation and a gantry rotation of $350 \mathrm{~ms}(\mathrm{~mA}=300$ to $800, \mathrm{kV}=120)$. Pitch (0.16 to 0.24$)$ was individualized to the patient's heart rate. The CCTA data sets were reconstructed with an increment of $0.4 \mathrm{~mm}$ using the cardiac phase with the least cardiac motion.

\section{CCTA image analysis}

Images were processed using the GE Advantage Volume Share Workstation (GE Healthcare, USA) and visually interpreted by two expert observers blinded to all clinical data. A 17 -segment model of the coronary arteries and four-point grading score (normal, mild [ $<50 \%$ ], moderate $[50 \%$ to $69 \%$, severe $[\geq 70 \%]$ ) was used for the evaluation of coronary stenosis; obstructive CAD was defined as coronary diameter stenosis $\geq 50 \%$.

\section{LBBB criteria}

LBBB was recorded on a 12-lead ECG and defined as set by the Criteria Committee of the New York Heart Association (15): QRS interval $120 \mathrm{~ms}$; slurred/notched wide and predominant $\mathrm{R}$ waves in leads I, aVL, V5 and V6; slurred/ notched and broad S waves in V1 and V2 with absent or small R waves; mid-conduction delay defined as notching or a plateau in the mid-QRS wave; ventricular activation time $50 \mathrm{~ms}$ at the onset of the QRS interval; M-shaped QRS variants with occasionally wide $\mathrm{R}$ waves in V5 and V6; no initial Q-wave over the left precordium; and absence of pre-excitation.

\section{Statistical analysis}

Statistical analysis was performed using SPSS version 13.0 (IBM Corporation Inc, USA) for Windows (Microsoft Corporation, USA). Continuous variables were expressed as mean $\pm \mathrm{SD}$, or median and range where appropriate, and categorical data were expressed as percentages. Student's $t$ test with Levene test for assessing homoscedasticity or Mann-Whitney $U$ test and $\chi^{2}$ test or Fisher's exact test were used to compare continuous and categorical variables between groups, respectively. All $\mathrm{P}$ values refer to two-tailed tests of significance. $\mathrm{P}<0.05$ was considered to be statistically significant.

\section{RESULTS}

Patients' baseline characteristics are reported in Table 1 . Of the 39 study patients, $22(56 \%)$ were female and the mean age was $63 \pm 13$ years; all patients had LBBB diagnosed using ECG and confirmed by a cardiac electrophysiologist. SPECT results showed 24 (61\%) patients had a reversible septal defect and 15 (39\%) had a fixed septal defect. An analysis of the CCTA findings revealed 27 (69\%) patients had a normal CCTA (normal coronary arteries or nonobstructive CAD) and 12 (31\%) had an abnormal CCTA result (>50\% luminal stenosis). There was no relationship between patterns of SPECT perfusion abnormalities (fixed or reversible defect) and CCTA results $(\mathrm{P}=0.323)$. 


\section{DISCUSSION}

The main finding in the present study was that approximately $70 \%$ of patients with LBBB and abnormal SPECT findings had normal CCTA findings. The presence of septal perfusion abnormalities in patients with LBBB is common (either reversible or fixed), but is not necessarily due to CAD. Normal CCTA is known to have excellent negative predictive value and acts as a filter for further cardiac testing or imaging.

Vasodilator SPECT is more accurate than exercise in excluding left anterior descending (LAD) CAD in patients with $\operatorname{LBBB}(16,17)$. However, false-positive results are still a concern. The severity of the septal perfusion or anterior wall defect does not provide enough additional information to identify LAD disease. Subsequently, invasive coronary angiography is necessary after a positive dipyridamole study to identify LAD disease as a major progressive factor in patients with LBBB. Although vasodilator SPECT is more specific than exercise, no randomized studies have been performed.

The exact prevalence of septal perfusion defects in patients with LBBB is unknown, but some studies (17), which used vasodilator SPECT imaging, reported decreased false-positive reversible septal rates, from $40 \%$ to $4 \%$. As seen in our study, both reversible and fixed defects can occur in patients with LBBB, although reversible defects are more common and neither abnormality is predictive for CCTA (18). The mechanism for the apparent defect has not been completely established but likely results from compromise of diastolic blood flow due to the delayed septal contraction. This compromise is accentuated by increasing heart rate and, thus, is less common when using vasodilator stress with dipyridamole or adenosine than with exercise stress (19). In a recent study, Koepfli et al (20) concluded that the apparent relative septal underperfusion during exercise in LBBB patients with normal coronary arteries is due to a lateral hyperperfusion rather than due to a manifest septal flow decrease. The fact that this phenomenon is inducible and reversible by short-term right ventricular pacing suggests a mainly functional (but not structural) alteration as the underlying mechanism of this finding. Subsequently, the authors suggested that quantification of myocardial blood flow using positron emission tomography may help avoid misinterpretation of septal perfusion defects in patients with LBBB (20).

At the present time, there are limited data in the literature regarding CCTA for patients with LBBB. Ghostine et al (21) reported that accuracy, sensitivity, specificity, positive predictive value and negative predictive value of 64-slice computed tomography for detecting significant stenosis was $97 \%, 72 \%, 99 \%, 91 \%$ and $97 \%$, respectively; postive and negative predictive values were $85 \%$ and $92 \%$, respectively, for detection of significant CAD (21). Two more studies reported that CCTA can be used as a noninvasive diagnostic method for patients with LBBB, to evaluate CAD $(22,23)$.

Our study is the first to investigate the role of CCTA in patients with LBBB and abnormal SPECT findings. Based on our data, approximately $70 \%$ of patients with LBBB and abnormal SPECT had normal CCTA findings. CAD can be excluded with high confidence and no further testing is needed for such patients based on the very high negative predictive value of CCTA. However, patients with abnormal CCTA will need further work-up, most likely invasive coronary angiography.

\section{Study limitations}

Our study had some limitations. First, it was conducted retrospectively and involved a relatively small number of patients. Based on inclusion and exclusion criteria, many patients were excluded, including patients with a history of CAD and patients with a time interval $>65$ days between SPECT and CCTA. Second, there was no correlation between CCTA and invasive coronary angiography. Nevertheless, abnormal CCTA ( $>50 \%$ stenosis) is considered to be abnormal in real clinical practice. We believe further prospective studies must be performed to investigate this challenging area.
TABLE 1

Patient characteristics $(n=39)$

\begin{tabular}{ll}
\hline Men & $17(44)$ \\
Women & $22(56)$ \\
Age, years & $63 \pm 13$ \\
Heart rate, beats $/ \mathrm{min}$ & $62 \pm 24$ \\
Body mass index, $\mathrm{kg} / \mathrm{m}^{2}$ & $32 \pm 6$ \\
Left ventricular ejection fraction, \% & $48 \pm 16$ \\
Hypertension & $14(35)$ \\
Diabetes & $19(49)$ \\
Smokers & $10(25)$ \\
Hypercholesterolemia & $16(41)$ \\
\hline Data presented as $n(\%)$ or mean $+\mathrm{SD}$ &
\end{tabular}

Data presented as $n$ (\%) or mean $\pm S D$

\section{CONCLUSION}

Abnormal SPECT results (either reversible or irreversible defects) are common in patients with LBBB. This does not necessarily indicate the presence of CAD. Approximately 70\% of patients with LBBB and abnormal SPECT have normal CCTA. We strongly believe CCTA must be considered the first noninvasive test in patients without history of CAD and LBBB. Normal CCTA excludes CAD with high confidence and further testing for CAD is not warranted. However, patients with abnormal CCTA will need more testing for CAD and, most likely, invasive coronary angiography.

\section{REFERENCES}

1. Fahy GJ, Pinski SL, Miller DP, et al. Natural history of isolated bundle branch block. Am J Cardiol 1996;77:1185-90.

2. Hesse B, Diaz LA, Snader CE, Blackstone EH, et al. Complete bundle branch block as an independent predictor of all-cause mortality. Am J Med 2001;110:253-9.

3. Schneider JF, Thomas HE Jr, Sorlie P, et al. Comparative features of newly acquired left and right bundle branch block in the general population: The Framingham study. Am J Cardiol 1981;47:931-40.

4. Ozdemir K, Altunkeser BB, Danis G, et al. Effect of the isolated left bundle branch block on systolic and diastolic functions of left ventricle. J Am Soc Echocardiogr 2001;14:1075-9.

5. McAnulty JH, Rahimtoola SH, Murphy E, et al. Natural history of "high-risk" bundle-branch block:final report of a prospective study. N Engl J Med 1982;307:137-43.

6. Nieman K, Cademartiri F, Lemos PA, et al. Reliable noninvasive coronary angiography with fast submillimeter multislice spiral computed tomography. Circulation 2002;106:2051-4.

7. Meijboom WB, Meijs MF, Schuijf JD, et al. Diagnostic accuracy of 64-slice computed tomography coronary angiography: A prospective, multicenter, multivendor study. J Am Coll Cardiol 2008;52:2135-44.

8. Kuettner A, Kopp AF, Schroeder S, et al. Diagnostic accuracy of multidetector computed tomography coronary angiography in patients with angiographically proven coronary artery disease. J Am Coll Cardiol 2004;43:831-9.

9. Garcia MJ, Lessick J, Hoffmann MH, CATSCAN Study Investigators. Accuracy of 16-row multidetector computed tomography for the assessment of coronary artery stenosis. JAMA 2006;296:403-11.

10. Kopp AF, Kuttner A, Trabold T, et al. Multislice CT in cardiac and coronary angiography. Br J Radiol 2004;77(Spec No 1):S87-S97.

11. Stillman AE, Oudkerk M, Ackerman M, et al.Usefulness of 64-slice multidetector computed tomography in diagnostic triage of patients with chest pain and negative or nondiagnostic exercise treadmill test result. Am J Cardiol 2007;99:925-9.

12. Stillman AE, Oudkerk M, Ackerman M, et al. Use of multidetector computed tomography for the assessment of acute chest pain: A consensus statement of the North American Society of Cardiac Imaging and the European Society of Cardiac Radiology. North American Society of Cardiac Imaging; European Society of Cardiac Radiology. Int J Cardiovasc Imaging 2007;23:415-27.

13. Van Train KF, Areeda J, Garcia EV, et al. Quantitative same-day rest-stress technetium-99m-sestamibi SPECT: Definition and validation of stress normal limits and criteria for abnormality. J Nucl Med 1993;34:1494-502. 
14. Cerqueira MD, Weissman NJ, Dilsizian V, et al. Standardized myocardial segmentation and nomenclature for tomographic imaging of the heart: A statement for healthcare professionals from the Cardiac Imaging Committee of the Council on Clinical Cardiology of the American Heart Association. J Nucl Cardiol 2002;9:240-5.

15. Criteria Committee of the New York Heart Association. Nomenclature and criteria for diagnosis of diseases of the heart and great vessels, 9th edn. Boston, MA: Little, Brown, 1994:210-9.

16. Lebtahi NE, Stauffer JC, Delaloye AB. Left bundle branch block and coronary artery disease: Accuracy of dipyridamole thallium-201 single-photon emission computed tomography in patients with exercise anteroseptal perfusion defects. J Nucl Cardiol 1997;4:266-73.

17. Iskandrian AE. Detecting coronary artery disease in left bundle branch block. J Am Coll Cardiol 2006;48:1935-7.

18. O'Keefe JH Jr, Bateman TM, Barnhart CS. Adenosine thallium-201 is superior to exercise thallium-201 for detecting coronary artery disease in patients with left bundle branch block. J Am Coll Cardiol 1993;21:1332.
19. Hansen CL. The conundrum of left bundle branch block. J Nucl Cardiol 2004;11:90-2.

20. Koepfli P, Wyss CA, Gaemperli O, et al. Left bundle branch block causes relative but not absolute septal underperfusion during exercise. Eur Heart J 2009;30:2993-9.

21. Ghostine S, Caussin C, Daoud B, et al. Non-invasive detection of coronary artery disease in patients with left bundle branch block using 64-slice computed tomography. J Am Coll Cardiol 2006;48:1929-34.

22. Nural MS, Yazici M, Elmali M, et al. The diagnostic value of multislice computed tomography in evaluation of coronary artery disease in patients with left bundle branch block. Anadolu Kardiyol Derg 2008;8:128-33.

23. Jankauskas A, Zaveckiene J, Unikas R, et al. Diagnostic value of multislice computed tomography coronary angiography in patients with left bundle branch block. Medicina (Kaunas) 2009;45:255-61. 